\title{
ORIGINAI
}

\section{LA TRANSICIÓN DEMOGRÁFICO-EPIDEMIOLÓGICA EN CHILE, 1960-2001}

\section{Jorge Szot Meza}

Departamento de Epidemiología. Ministerio de Salud. Santiago de Chile.

\section{RESUMEN}

Fundamento: En Chile se ha modificado de manera significativa la estructura demográfica y las causas de morbilidad y de mortalidad de la población en un período relativamente breve de tiempo. Estas variaciones se enmarcan dentro de los procesos denominados transición demográfica y transición epidemiológica respectivamente. El propósito de este trabajo es evidenciar la transición demográfica y la transición epidemiológica ocurridas en el país entre 1960 y 2001

Método: Se trata de un estudio de series temporales que recoge información demográfica y de mortalidad de Chile entre los años 1960 y 2001. Los datos fueron obtenidos de los Anuarios de Demografía del Instituto Nacional de Estadísticas (INE), de información producida por el Departamento de Estudios Prospectivos del Ministerio de Planificación y Cooperación y del Ministerio de Salud de terio de
Chile.

Resultados: En 1960 un 39,6\% de la población tenía menos de 14 años, la tasa de natalidad era de 36,3 por 1000 , la tasa de mortalidad general de 12,3 por 1.000 y la tasa de mortalidad infantil de 120 por 1.000 . El $44 \%$ de la mortalidad general se generaba en causas infecciosas y perinatales. En el año 2001 un $28 \%$ de la población tiene menos de 14 años, la tasa de natalidad es de 18,3 por 1.000 , la tasa de mortalidad general de 5,4 por 1.000 y la tasa de mortalidad infantil de 8,9 por 1000 . El $68 \%$ de la mortalidad general se produce por enfermedades crónicas.

Conclusiones: En base a los resultados, es posible afirmar que Chile se encuentra en una fase tardía tanto de transición demográfica como de transición epidemiológica. Conocer la realidad de Chile aportará información a aquellos países de América Latina que se encuentren en fases más tempranas de este tipo de transiciones.

Palabras clave: Transición demográfica. Transición epidemiológica. Chile. Tasa de natalidad. Tasa de mortalidad. Mortalidad perinatal.

Correspondencia:

Ezequías Alliende 2352, depto. 604

6640000 Providencia

Santiago de Chile.

Correo electrónico: jszot@minsal.gov.c

\section{ABSTRACT}

\section{The Demographic-Epidemiological Transition in Chile, 1960-2001}

Background: In Chile, the demographic structure and the causes of morbidity and of mortality among the population have undergone major changes within a relatively short period of time. These changes have taken place within a framework of processes referred to respectively as demographic transition and epidemiological transition. This study is aimed at providing evidence as to the demographic transition and epidemiological transition which took place in this country within the 1960-2001 period.

Methods: This is a time-series study gathering demographic and death rate-related information from Chile throughout the 1960-2001 period. The data was taken from the Demographic Yearbooks of the National Institute of Statistics, from information produced by the Department of Prospective Studies under the Ministry of Planning and Cooperation and the Chilean Ministry of Health.

Results: In 1960, 39,6\% of the population had less than 14 years old, natality rate was 36,3 per 1000 , general mortality rate 12,3 per 1000 and Infant Mortality rate 120 per $1000.44 \%$ of causes of deceases were by infectious and newborn's diseases. In 2001, $28 \%$ of the population has less than 14 years old, natality rate is 18,3 per 1000, General Mortality rate 5,4 per 1000 and Infant Mortality rate 8,9 per $1000.68 \%$ of causes of deceases are by chronic diseases.

Conclusions: Based on the results, it can be said that Chile is currently in a late stage of demographic transition as well as epidemiological transition. Ascertaining Chile's actual situation will promiological transition. Ascertaining Chile's actual situation will pro-
vide information to those Latin American countries which are vide information to those Latin American countries
currently in early stages of these types of transitions.

Key words: Demographic transition. Epidemiological transition,. Chile. Birth rate. Mortality rate. Perinatal mortality. 


\section{INTRODUCCIÓN}

Chile está viviendo desde la década de 1960 un proceso continuo de cambio desde el punto de vista de la estructura de su población y de las causas de enfermedad y muerte en ella ${ }^{1-3}$. Estos cambios, descritos en numerosos países, se denominan Transición Demográfica (TD) y Transición Epidemiológica (TE) respectivamente.

La TD fue descrita por Omram ${ }^{4}$ a comienzos de los años 70 y corresponde a un proceso de evolución de las características demográficas de la población, que pasa desde un estado inicial (Fase Temprana de la TD) caracterizado por una alta tasa de natalidad (TN) y una alta tasa de mortalidad general (TMG), a un estado posterior (Fase Tardía de la TD) en que caen las TN y $\mathrm{TMG}^{5-7}$. Como consecuencia de lo anterior, la población, que en un comienzo está constituida principalmente por personas jóvenes (alto número de nacimientos y muertes ocurridas a edades precoces) en la fase tardía está constituida principalmente por adultos y adultos mayores (desciende el número de nacimientos y las muertes ocurren a edades más avanzadas).

La TE es un concepto que complementa el anterior y que fue acuñado por Frenck ${ }^{8}$. Se refiere a la modificación en las causas de enfermedad y muerte en la población conforme se modifica su estructura demográfica. La TE también evoluciona en fases, y éstas coinciden con las fases de evolución que se han descrito para la TD. Así, en la fase temprana de la TE, cuando predomina la población joven, estos enferman y fallecen principalmente de enfermedades infecciosas (EI) y traumatismos, envenenamientos y violencia (TEV). En la fase tardía de la TE, al vivir las personas un mayor número de años, se encuentran, durante un periodo de tiempo más prolongado, bajo la influencia de los factores de riesgo para enfermedades crónicas, determinando que sean éstas entonces sus principales causas de morbilidad y mortali$\operatorname{dad}^{9,10}$.
Desde el punto de vista de la existencia de estos procesos en América Latina, tanto la TD como la TE afectan a los países de manera diferente ${ }^{11-13}$. Así mientras el Cono Sur (Argentina, Uruguay y Chile) y Cuba se encuentran en una fase avanzada de TD y TE (11), en el extremo opuesto países como Honduras, Guatemala y Bolivia se encuentran atravesando etapas tempranas tanto de TD como de TE (14). La gran mayoría de los países de América Latina, sin embargo, se encuentra en etapas intermedias de ambos $\operatorname{procesos}^{11}$.

El propósito del presente trabajo, es mostrar el proceso de TD y TE que se ha presentado en Chile a partir de la década de los años 60 .

\section{MATERIAL Y MÉTODO}

Se trata de un Estudio de Serie Temporal, que recoge información demográfica y de mortalidad de Chile entre los años 1960 y 2001. Los datos fueron obtenidos de los Anuarios de Demografía del Instituto Nacional de Estadísticas (INE), de información producida por el Departamento de Estudios Prospectivos del Ministerio de Planificación y Cooperación y del Ministerio de Salud de Chile y se presentan de la siguiente forma:

1. Información Demográfica (para 1960 y 2001): distribución de la población según tramos quinquenales de edad (construcción Pirámides de Población) y distribución por grandes grupos de edad; de 0 a 14 años, de 15 a 64 años y mayores de 65 años, Tasa de Natalidad (TN: $\mathrm{N}^{\circ}$ de nacidos vivos en un año / población total al 30 de junio de ese año x 1000), Tasa de Mortalidad General (TMG: $\mathrm{N}^{\mathrm{o}}$ total de defunciones en un año / población total al 30 de junio de ese año x 1000) y Crecimiento Vegetativo $\left(\mathrm{N}^{\circ}\right.$ de nacidos vivos en un año / población total al 30 de junio de ese año x 100).

Rev Esp Salud Pública 2003, Vol. 77, N. ${ }^{\circ} 5$ 
2. Información de Mortalidad por grupos de causas (para 1960 y 1999 respectivamente): Proporción de muerte por grandes grupos de causas, clasificadas de acuerdo a la Séptima Revisión de la Clasificación Internacional de Enfermedades y Estados Mórbidos (CIEVII) (15) para 1960 y a la CIE-X en $1999^{16}$.

3. Información de Mortalidad Infantil (1960-2000): tendencia de la Tasa de Mortalidad Infantil $\left(\mathrm{N}^{\circ}\right.$ defunciones en menores de un año / $\mathrm{N}^{\circ}$ nacidos vivos en un año $x$ 1000) y causas de defunción en menores de 1 año según la CIE-VII para 1960 y la CIE-X en 2000 .

\section{RESULTADOS}

Los resultados se presentan en forma de tablas y figuras. Las Pirámides de Población de Chile de los años 1960 y 2001 se presentan en la figura 1. En ésta se aprecia como la representación gráfica de la distribución de población del país ha pasado de ser «piramidal», con una base amplia que representa a los grupos de edad más jóvenes, a una distribución «cónica», en la que los segmentos de población adulta y adulta-mayor han adqui- rido una mayor importancia relativa. Los indicadores demográficos como tasa de natalidad, tasa de mortalidad general y crecimiento vegetativo, para el periodo comprendido entre los años 1960 y 1999 se muestran en las figuras 2, 3 y 4 respectivamente, observándose el descenso de todas ellas, aunque con una tendencia esperable al estancamiento en la disminución de la mortalidad general, la que debiera comenzar a aumentar en los próximos años conforme continúe el proceso de envejecimiento de la población. La tabla 1 compara la distribución de población por grandes grupos de edad para Chile, entre 1960 y 2001, destacando el hecho del aumento de la población mayor de 65 años de edad. Las principales causas de mortalidad general para 1960 y 1999 se observan en la tabla 2. Se observa como las enfermedades infecciosas y perinatales, que ocasionaban un $44 \%$ de las defunciones en 1960, han sido sustituidas por las enfermedades cardiovasculares y las neoplasias, que produjeron un $50 \%$ de las defunciones en 1999. La tendencia y grupos de causas de mortalidad infantil, entre 1960 y 2000, se muestran en la figura 5 y la tabla 3, respectivamente. Aquí es posible observar la enorme diminución de la TMI y la modificación en la composición de las causas de muerte en los menores de 1 año.

Figura 1

Pirámides de población de Chile, 1960 y 2001
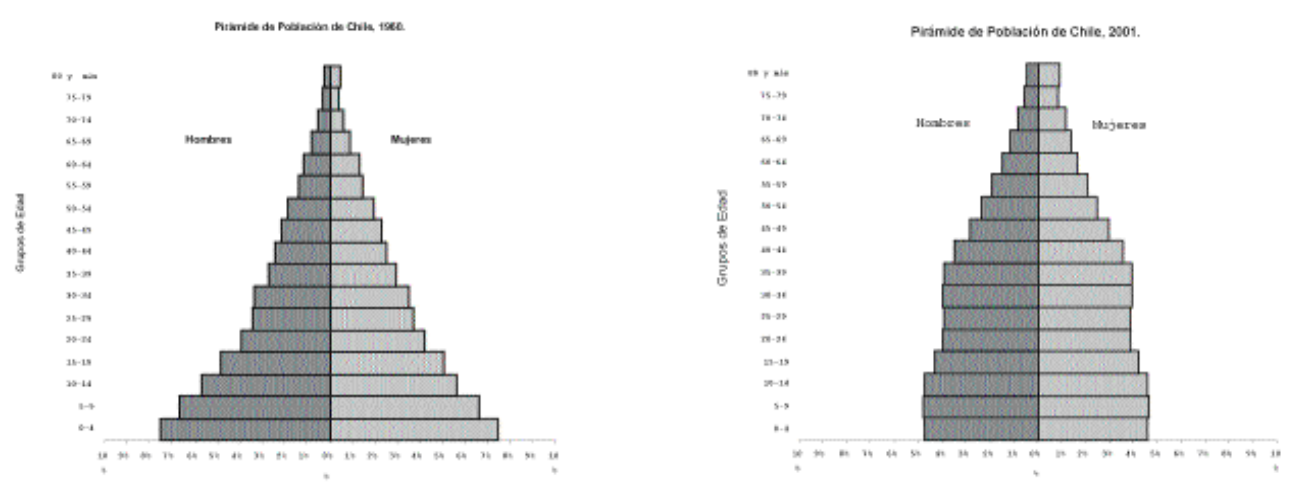
Figura 2

Evolución de la tasa de natalidad. Chile, 1960-1999

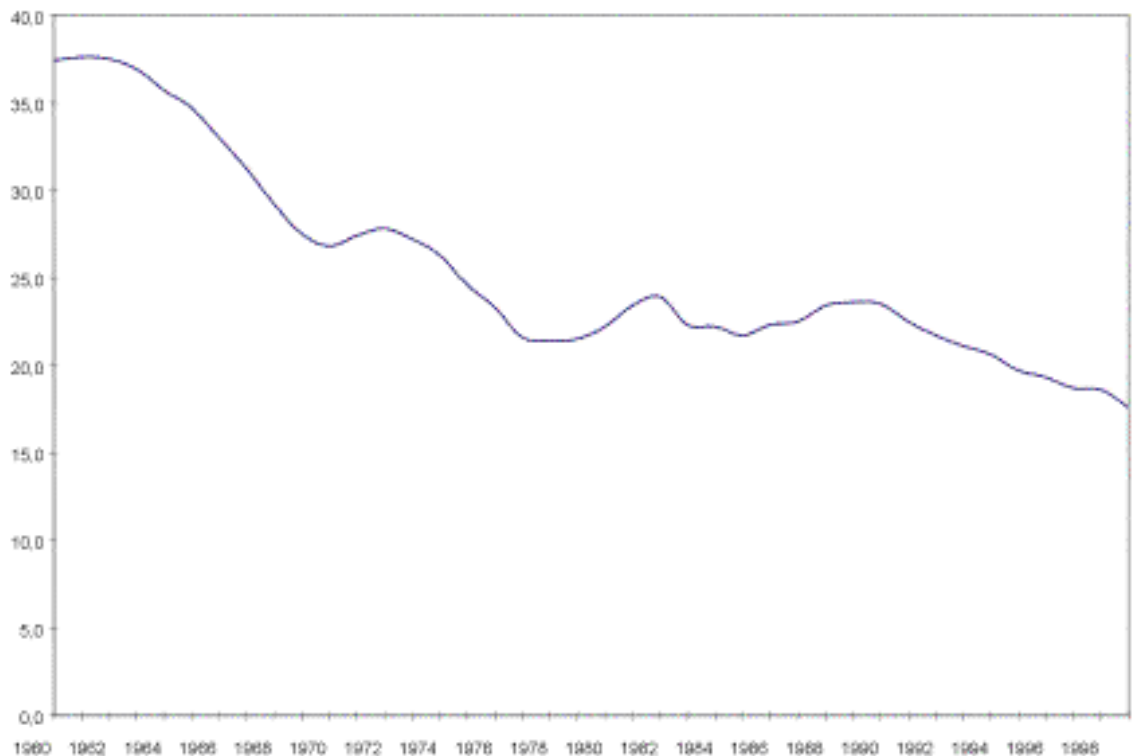

Añ̄o

Figura 3

Evolución de la tasa de mortalidad general. Chile, 1960-1999

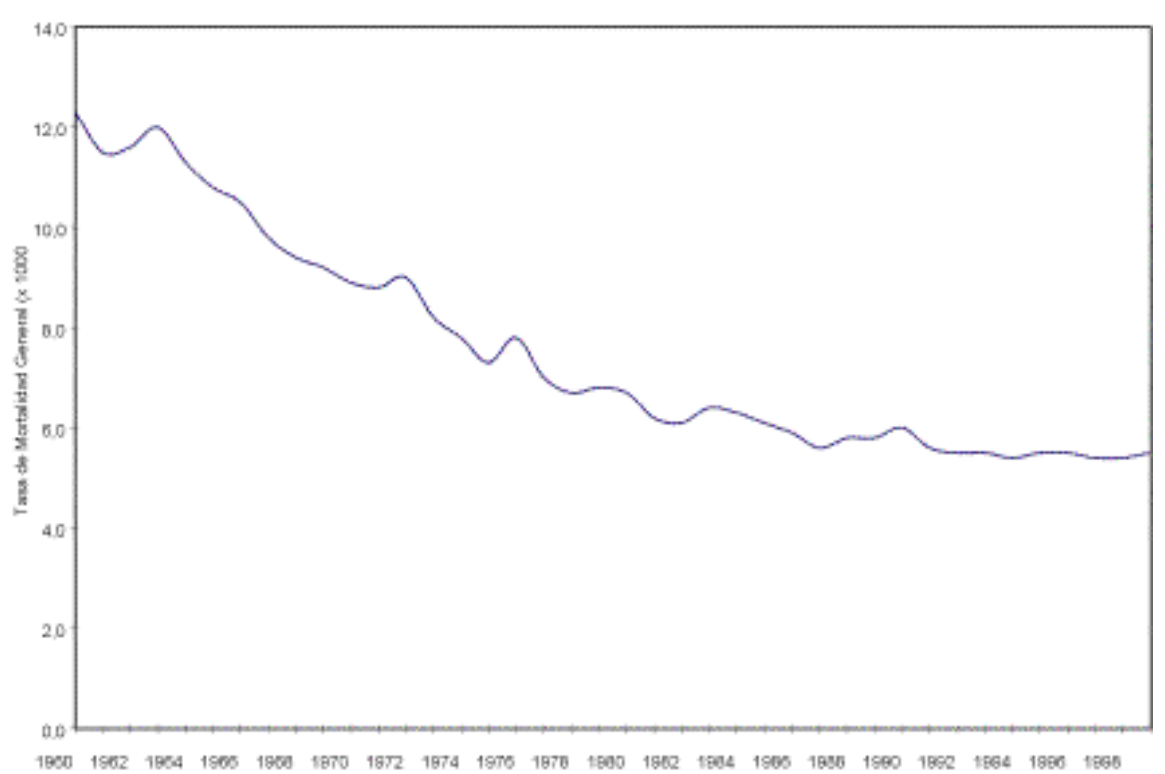


Figura 4

Evolución del crecimiento vegetativo. Chile, 1960-1999

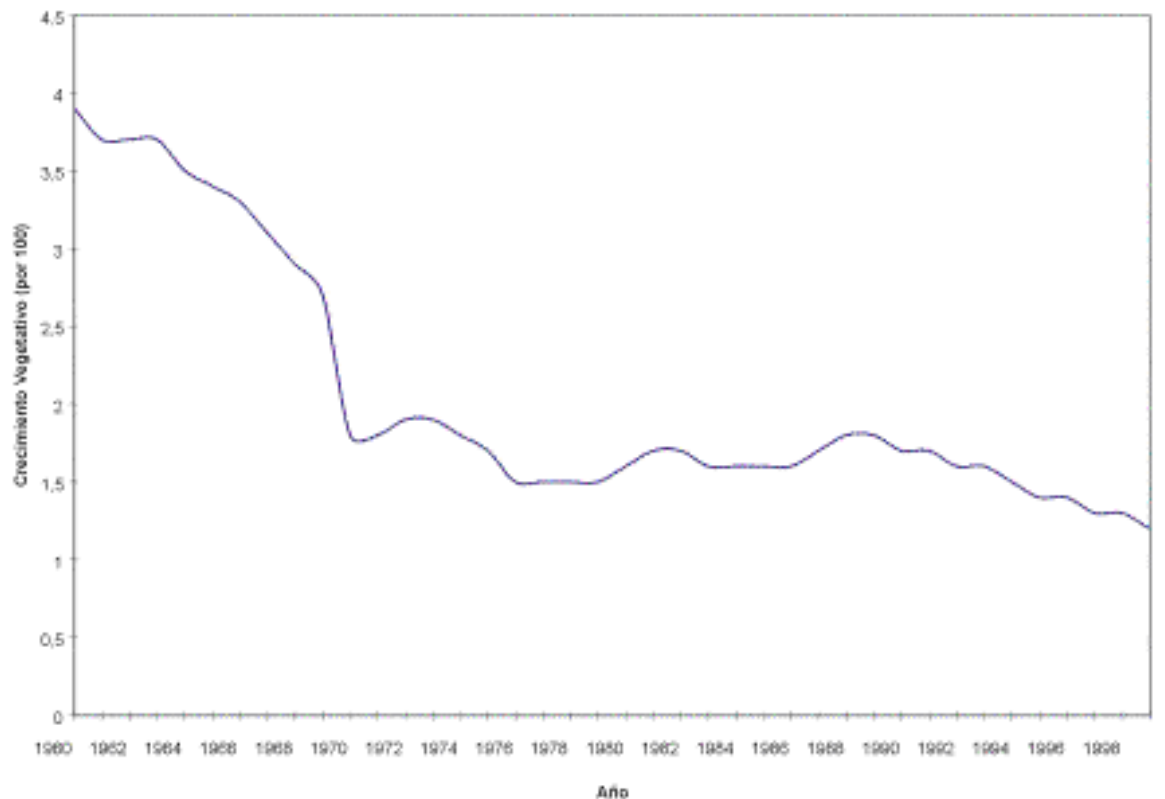

Tabla 1

Distribución de frecuencia de la población general, Chile, 1960-2001

\begin{tabular}{|ccc|}
\hline Grupo de edad (afous) & $1960(\%)$ & $2001(\%)$ \\
\hline $0=14$ & 39,6 & 28,0 \\
$15-64$ & 56,0 & 64,7 \\
$65 y$ mis & 4,3 & 7,3 \\
TOTAL & 100 & 100 \\
\hline
\end{tabular}

\section{DISCUSIÓN}

Desde el punto de vista de la estructura de la población chilena se ha producido un cambio notable. En 1960 los habitantes menores de 15 años constituían el 39,6\% de la población y los mayores de 65 años un 4,3\%. En el año 2001, la población menor de
15 años correspondió al $28 \%$ y los mayores de 65 años alcanzaban el 7,3\%

Esta modificación de las características de la población del país, se debe a múltiples factores: ha disminuido la TN, ha bajado la TMG y ha descendido el CV. En 1960, la TN era de 36,3 por 1000 habitantes y la TMG de 
Tabla 2

Distribución de frecuencia de causas de muertes según grandes grupos de causas en ambos sexos (CIE-VII), Chile, 1960 y 1999

\begin{tabular}{|lcc|}
\hline Cuusa & $\begin{array}{c}1960 \\
\text { (CIE VII) }\end{array}$ & $\begin{array}{c}1999 \\
\text { (CIE X) }\end{array}$ \\
\hline Peripe, neumonia & $15 \%$ & - \\
Infecciosas & $20 \%$ & - \\
Mal definidas & $9 \%$ & $5 \%$ \\
Tumores malignos & $9 \%$ & $23 \%$ \\
Traumas & $8 \%$ & $9 \%$ \\
Otras & $7 \%$ & $11 \%$ \\
Enfermedades del sistema circulatorio & $25 \%$ & $27 \%$ \\
Enfermedades endocrinas & $7 \%$ & $4 \%$ \\
Enfermedades del sistema digestivo & - & $7 \%$ \\
Enfermedades del sistema respiratorio & - & $14 \%$ \\
\hline Total & $100 \%$ & $100 \%$ \\
\hline
\end{tabular}

* Incluye: Tuberculosis, Sífilis, Fiebre Tifoidea, Disentería, Escarlatina, Difteria, Tos ferina, Infección meningocócica, Poliomielitis, Sarampión, Tifus, Fiebre Reumática

12,3 por 1000 habitantes. En el año 2001, La TN y la TMG registran valores de 18,3 por 1000 habitantes y de 5,4 por 1000 habitantes respectivamente 17.

A continuación se analizarán con mayor detalle los cambios que se han producido en estos indicadores.

La considerable disminución que ha experimentado en Chile la TN obedece, por una parte, a la instauración en el país, a mediados de la década de los años 60, de la Política Nacional de Regulación de la Fecundidad y por otro lado a la reducción de la Tasa Global de Fecundidad $(18,19)$ (TGF: número de hijos por mujer al culminar su edad reproductiva). La reducción en la TGF es a su vez consecuencia de un mejor nivel de instrucción de la madre, la que generalmente opta por proseguir sus estudios, o incorporarse al mercado laboral, lo que se vería dificultado si su paridad fuese elevada.

La TMG se ha reducido a menos de la mitad del valor registrado en 1960 gracias al mejoramiento de las condiciones generales de vida de la población y en gran medida al marcado descenso de la TMI en Chile.

La disminución del CV se explica porque mientras la población general del país ha 
Figura 5

Evolución de la Tasa de mortalidad infantil (TMI), Chile, 1960-2000

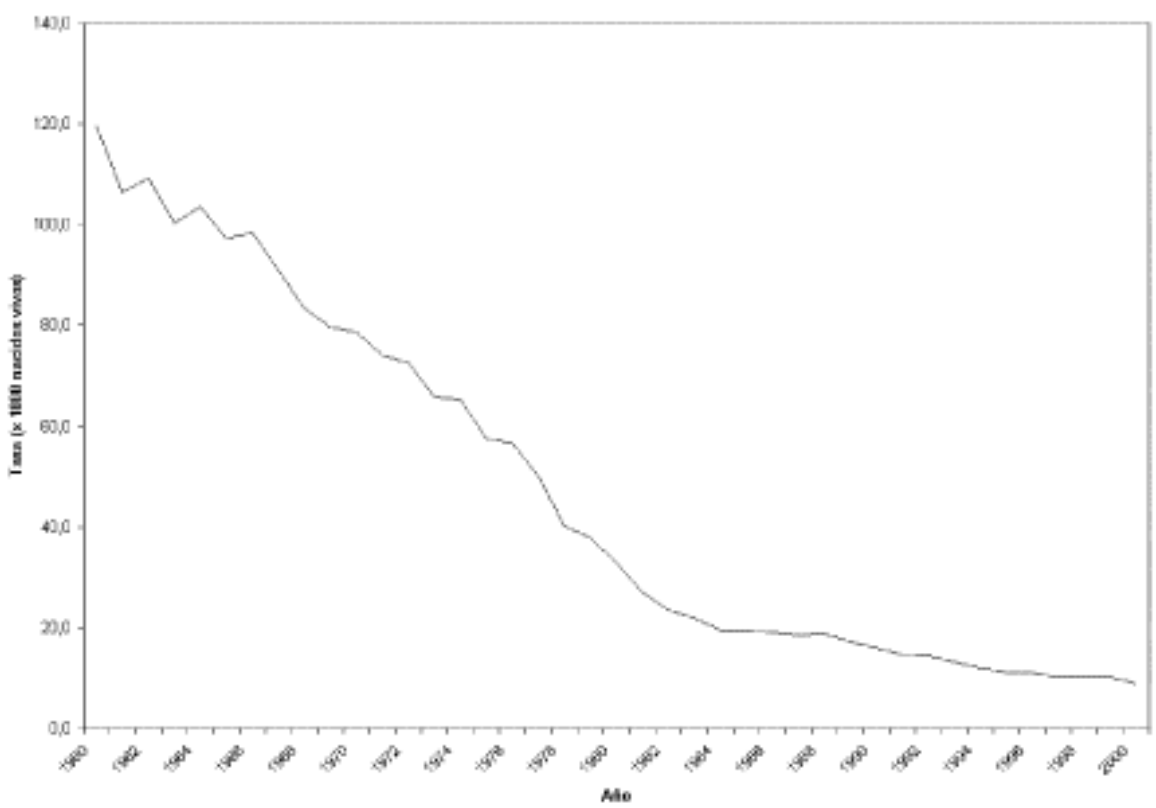

Tabla 3

Distribución de Frecuencia de las principales causas de mortalidad infantil, Chile, 1960 y 1999

\begin{tabular}{|c|c|c|}
\hline Causas & 1960 (CIE VII) & 1999 (CIE X) \\
\hline Neumonia & $28 \%$ & - \\
\hline Prematuridad & $27 \%$ & - \\
\hline Infeociones recién nacidos & $10 \%$ & - \\
\hline Enterocolitis & $10 \%$ & - \\
\hline Lesiones debidas al parto & $5 \%$ & - \\
\hline Infoociosas* & $5 \%$ & $3^{n} \%$ \\
\hline Malformaciones & $2^{\circ}$ & $32 \%$ \\
\hline Traumatismos, envenenamentos y violencia & $=$ & $5^{\circ} \%$ \\
\hline Respiratorias & - & $12 \%$ \\
\hline Perinatales & - & $34^{\circ} \mathrm{c}$ \\
\hline Mal definidas & $4 \%$ & $\cdot$ \\
\hline Otras & $9 \%$ & $14 \%$ \\
\hline TOTAL & $100 \%$ & $100 \%$ \\
\hline
\end{tabular}

* Incluye: Tuberculosis, Sífilis, Fiebre Tifoidea, Disentería, Escarlatina, Difteria, Tos ferina, Infección meningocócica, Poliomielitis, Sarampión, Tifus, Fiebre Reumática.

Rev Esp Salud Pública 2003, Vol. 77, N. ${ }^{\circ} 5$ 
aumentado, el número absoluto de nacimientos se ha mantenido en un nivel relativamente constante, en torno a los 250.000 nacimientos al año ${ }^{17}$.

Estos cambios demográficos, de acuerdo a la Teoría descrita por Omram sitúan a Chile en la fase tardía de la TD.

Ahora bien, como es de suponer, los cambios demográficos se han traducido en cambios epidemiológicos. Mientras en 1960 las enfermedades infecciosas ocasionaban en Chile un $30 \%$ de las defunciones, esta cifra se había reducido a un $3,0 \%$ en $1999^{20}$. De forma opuesta, las enfermedades crónicas no transmisibles del adulto (ECNTA) excluyendo a las enfermedades respiratorias y los traumatismos, envenenamientos y violencias (TEV) que originaban un $14 \%$ de las muertes en 1960 produjeron un 68,2\% de ellas en $1999^{20}$. Los datos expuestos evidencian entonces, de acuerdo a lo postulado por Frenck, que el país se encuentra cursando la fase tardía de la TE, en coincidencia, como se señalaba, con la fase tardía de TD.

Mención especial merece el caso de disminución de la mortalidad infantil en el país, que ha comienzos de la década del 60 tenía en Chile una de las cifras más elevadas de América Latina: 34.314 defunciones en menores de 1 año, con una TMI de 120 por 1000 nacidos vivos, cifra que ha bajado 2.336 defunciones en menores de 1 año con una TMI de 8.9 por 1000 nacidos vivos en el año $2000^{21}$. Las causas son múltiples, algunas de las cuales ya se han mencionado: como la disminución de la TN, de la TGF y gracias al mejoramiento de las condiciones generales de vida de la población, así como al mayor nivel de instrucción de la madre.

Pero no solo ha cambiado el indicador, la etiología de las defunciones en el menor de 1 año también se han modificado. Mientras en 1960 la TMI estaba dada principalmente por causas infecciosas, en 1999 las muertes se producían por malformaciones congénitas y alteraciones cromosómicas y afecciones perinatales asociadas con la prematuridad extrema ${ }^{20}$.

Puede plantearse entonces que, de mantenerse en Chile la tendencia demográfica actual, y sin la existencia de fenómenos migratorios de importancia, el país se verá enfrentado durante los próximos años a un progresivo envejecimiento de su población, con el consiguiente aumento de la TMG. También es esperable mantener la tendencia decreciente de la TN y de la TMI. Desde el punto de vista de la morbilidad en la población, cabe esperar una mayor incidencia de enfermedades emergentes y re-emergentes (como la neumonía del adulto mayor o la tuberculosis en pacientes portadores del Virus de Inmunodeficiencia Humana). Este proceso se denomina de Post-transición Demográfica, y es el que están viviendo la mayoría de las sociedades industrializadas en el mundo occidental.

La situación demográfica y epidemiológica chilena, similar a la que presenta un grupo de países de América Latina, puede servir como referencia a los países que se encuentran en etapas más precoces de TD y TE de tal forma que éstos conozcan algunos aspectos de la realidad sanitaria que deberán enfrentar en el mediano plazo.

\section{BIBLIOGRAFÍA}

1. Albala C, Vio F, Robledo A, Icaza G. La Transición Epidemiológica en Chile. Rev Med Chile 1993;121:1446-55.

2. Albala C, Vio F, Yáñez M. Transición Epidemiológica en América Latina: Comparación de Cuatro Países. Rev Med Chile 1997;125:719-27.

3. Szot J, Moreno C. Transición Demográfica y Epidemiológica en el Servicio de Salud Metropolitano Sur Oriente. Cuad Med Soc 2000;41:21-7.

4. Omran R. The Epidemiological Transition: a Theory of the Epidemiology of the Population Change. Milbank Mem Fund Q 1971;49:509-38.

Rev Esp Salud Pública 2003, Vol. 77, N. ${ }^{\circ} 5$ 
5. Vio F, Albala C. Nutrition Policy in the Chilean Transition. Public Health Nutr 2000;3:49-55.

6. Wall S. Epidemiology in Transition. Int J Epidemiol 1999;28:1000-4.

7. Thelle S. The Epidemiological Transition During the 20th Century. Tidsskr Nor Laegeforen 1999;119:4494-6.

8. Frenck J, Frejka T, Bobadilla J, Stern C, Lozano R, Sepúlveda J. La Transición Epidemiológica en América Latina. Bol Oficina Sanit Panam 1991:111:485-96.

9. Berríos X, Jadue L, Centeno J. Prevalencia de Factores de Riesgo de Enfermedades Crónicas. Estudio en la Población General de la Región Metropolitana, 1986-1987. Rev Med Chile 1990:118:597604.

10. Berríos X, Szot J, Guzmán B, Cifuentes M. Homocisteína Plasmática: un Nuevo Factor de Riesgo para Enfermedad Coronaria. Su exploración en una Población Escolar, Región Metropolitana, Chile. Rev Chil Cardiol 2000; 19: 43-50.

11. Castillo C, Mújica O, Loyola E. A Subregional Assessment of the Demographic and Health Trends in the Americas: 1980-1998. Stat Bull Metrop Insur Co 1999;80:2-12.

12. Mazzafero V, Masse G. La Transición Demoepidemiológica en Europa y América Latina. Rev Inst Hig Med Soc 1999;3:9-20.
13. Ministerio de Salud. Memoria de la Cooperación Externa 1997. Managua: Ministerio de Salud; 1998.

14. Instituto Nacional de Estadística. La Transición de la Fecundidad en Bolivia y sus Determinantes (1965-1995). La Paz: Instituto Nacional de Estadística; 1997.

15. Séptima Revisión Internacional de Enfermedades y Estados Mórbidos. Washington: Organización Panamericana de la Salud; 1955.

16. Décima Revisión Internacional de Enfermedades y Estados Mórbidos. Washington: Organización Panamericana de la Salud; 1995.

17. Instituto Nacional de Estadísticas (INE). Anuario de Demografía 1999. Santiago de Chile; 2000.

18. Szot J. Mortalidad infantil e indicadores económicos en Chile: 1985-1999, Rev Med Chile 2002;130:107-12.

19. Rosselot E, Meneguello J, Salud de la Familia y Paternidad Responsable, La Experiencia de Chile, 1965-1988. Rev Med Chile 1990, 118: 330-338.

20. Ministerio de Salud Estadísticas de Natalidad y Mortalidad 1999. Santiago de Chile: Ministerio de Salud; 2000.

21. Instituto Nacional de Estadísticas (INE). Anuario de Demografía 2000. Santiago de Chile; 2002. 